\title{
Study on the isolation of active constituents in Lonicera japonica and the mechanism of their anti-upper respiratory tract infection action in children
}

\author{
Hongxia Lu, Lei Zhang, Han Huang
}

\begin{abstract}
The Second Branch of Respiration Department of Zhengzhou Children's Hospital of Henan Province, Zhengzhou, 450053, China
\end{abstract}

\begin{abstract}
Background: Lonicera japonica has been studied extensively by scholars at home and abroad, a number of compounds have been isolated from it, which mainly include organic acids and flavonoids. Pharmacological studies have shown that Lonicera japonica has antibacterial and gall bladder-protective effects.

Objective: To study the active constituents in Lonicera japonica and the mechanism of their anti-upper respiratory tract infection action in children.

Methods: Compounds were identified by chromatographic methods, and the mechanism of anti-pediatric upper respiratory tract infection action of Lonicera japonica decoction was studied using experimental animals.

Results: A total of four compounds were isolated, after injection of egg white, toe edema in rats in the control group was very obvious, different test concentrations of Lonicera japonica decoction all inhibited toe edema in rats to some extents, the edema was the mildest in the Lonicera japonica decoction high-dose group, which had the strongest inhibitory effect on the development of inflammation, the Lonicera japonica decoction showed certain dose-effect relationship with toe edema in rats. In the rat body temperature control experiment, while body temperature of rats in the blank group had already risen, other groups were still able to lower the body temperature of rats under the action of test drugs. The severity of ear edema in mice in the blank control group was obvious, with increased thickness which showed significant difference between left and right ears. Under test doses, three Lonicera japonica decoction groups all inhibited xylene-induced ear edema in mice.

Conclusion: Lonicera japonica has an anti-upper respiratory tract infection action in children.

Keywords: Lonicera japonica, chlorogenic acid butyl ester, oleanolic acid, mouse ear edema

DOI: http://dx.doi.org/10.4314/ahs.v15i4.32

Cite as: $L u H$, Zhang L, Huang H. Study on the isolation of active constituents in Lonicera japonica and the mechanism of their anti-upper respiratory tract infection action in children. Afri Health Sci. 2015;15(4):1295-301. http://dx.doi.org/10.4314/ abs.v15i4.32
\end{abstract}

\section{Introduction}

Lonicera japonica is the dried bud or the flower of initial blooming of Lonicera japonica Thunb. in the family Caprifoliaceae, it is sweet in taste, cold in nature, and has the actions of clearing away heat and toxic materials and relieving wind-heat, which is mainly used in the treatment of carbuncle, furunculosis, pharyngitis, erysipelas, bloody flux, common wind-heat cold, febrile disease, fever, etc. ${ }^{1}$ As a common Chinese medicinal herb, Lonicera japonica has been studied extensively by scholars at home and abroad, a number of compounds

\footnotetext{
Corresponding author:

Hongxia Lu,

The Second Branch of Respiration

Department of Zhengzhou Children's Hospital of Henan Province, Zhengzhou, 450053, China

E-mail: doctorluhongxia@126.com
}

have been isolated from it, which mainly include organic acids, flavonoids, triterpenoid saponins and volatile oils $^{2-7}$. Pharmacological studies have shown that Lonicera japonica has antibacterial, antiviral, anti-inflammatory, antipyretic, liver and gall bladder-protective, anti-oxidative, anti-fertility, anti-platelet aggregation, hypolipidemic, anti-allergic and immunoregulatory effects $^{8-12}$.

To clarify the active constituents in Lonicera japonica, and investigate the mechanism of anti-pediatric upper respiratory tract infection action of Lonicera japonica, in this paper, the active constituents in Lonicera japonica were isolated and purified, and their structures were identified. Meanwhile, the anti-inflammatory effect of Lonicera japonica decoction was studied using experimental animal model.

\section{Methods}

BRUKER AV600 NMR spectrometer (600HZ); Koffiler micro melting point apparatus; ESI- Ms (Finnigan, 
USA); electrospray ionization (ESI) source; column chromatography silica gel (200-300 mesh), Qingdao Haiyang Chemical Plant .

$4 \mathrm{Kg}$ of dried Lonicera japonica was taken, and extracted separately with an 8-fold amount of ethanol (95\%) three times, each time lasted $2 \mathrm{~h}$. The ethanol extracts were combined, and ethanol was evaporated to give Lonicera japonica ethanol extractum.

The extractum was dissolved in distilled water, and extracted successively with petroleum ether, ethyl acetate and n-butanol three times, respectively, extractants were evaporated, then the petroleum ether and ethyl acetate extracts were taken and subjected separately to silica gel column chromatography, and gradient-eluted with petroleum ether-acetone or chloroform-methanol system as eluent, after repeated silica gel column chromatography, ODS column chromatography and preparative chromatography, four compounds were isolated.

Experimental study on the inhibition of pediatric upper respiratory tract infection by Lonicera japonica decoction

Wistar rats, 200-220 g, Kunming mice, 18-22 g, half male and half female, were purchased from China Medical University. Adaptive feeding was continued for weeks, and the rats in each group were fasted for $18 \mathrm{~h}$ before experiment.

Dried Lonicera japonica was taken, and decocted with a 6 -fold amount of water three times, decoction times were $1,0.5$ and $0.5 \mathrm{~h}$, respectively, the water decoctions were then combined, and concentrated to $1.2 \mathrm{~g} / \mathrm{mL}$ for later use. Dexamethasone acetate cream (999 Pi Yan Ping), xylene, dry yeast, dexamethasone acetate tablets.

\section{Definition}

50 rats whose body temperature was in a normal state were selected, and randomly divided into five groups, Lonicera japonica decoction treatment was administered intragastrically at 60,30 and $15 \mathrm{~g} / \mathrm{Kg}$, respectively, in the treatment groups. Aspirin was administered intragastrically at $0.3 \mathrm{~g} / \mathrm{Kg}$, in the asprin control group and the blank control group was given intragastrically an equivalent amount of distilled water. The rats were administered with the above, once daily for 6 consecutive days. On the 6th day after administration, the rats were immediately injected with $3 \mathrm{ml}$ of yeast solution (concentration of 20\%) at dorsal skin, after inducing pyrexia, body temperature of the rats was measured.

40 Kunming mice were taken, and randomly divided into four groups, namely the control group and the Lonicera japonica decoction treatment groups (dose as above). The decoction was administered once daily for 5 consecutive days. On the 5 th day, $1 \mathrm{~h}$ after administration, xylene was applied to left ear of mice to induce inflammation, $30 \mathrm{~min}$ later, the mice were sacrificed, left and right ears of the same sites were punched with a puncher and weighed on an electronic balance. The masses of the left and right ear pieces were recorded, and degree of edema was calculated.

Methods were the same as in 1,2,3. On the 5 th day after intragastric administration, $0.2 \mathrm{ml}$ of $10 \%$ fresh egg white was injected immediately in the left toe, toe thicknesses before and after egg white injection were measured, and D-value of edema was calculated.

\section{Statistical analysis}

Data were expressed as $\mathbf{X} \pm \mathbf{S}$, multi-sample comparison was performed by one-way ANOVA, and comparison among groups was performed by t test, statistical analysis was done using SPSS 15.0 statistical software.

\section{Results}

Structural identification of active constituents in Lonicera japonica

\section{Compound 1}

Yellow powder, 1H-NMR (300MHZ) $\delta: 1.08(3 \mathrm{H}, \mathrm{d}$, $\mathrm{J}=6.0 \mathrm{~Hz}, \mathrm{H}-6$ "'), 4.41 (1H, brs, H-1"'), $5.40(1 \mathrm{H}, \mathrm{d}$, $\mathrm{J}=7.0 \mathrm{~Hz}, \mathrm{H}-1$ "), $6.26(1 \mathrm{H}, \mathrm{d}, \mathrm{J}=1.8 \mathrm{~Hz}, \mathrm{H}-6), 6.43(1 \mathrm{H}$, d, J=1.8 Hz, H-8), $6.87\left(1 \mathrm{H}, \mathrm{d}, \mathrm{J}=8.5 \mathrm{~Hz}, \mathrm{H}-5^{\prime}\right), 7.58$ (IH, H-2'), 7.67 (1H, br.d, J=8.7 Hz, H-6'), $12.54(1 \mathrm{H}$, s, 5-OH); 13C-NMR (ppm) $\delta: 18.3$ (C-6”"), 67.7(C-6”), 68.7 (C-5"'), 70.2 (C-4"), 70.5 (C-2"'), 70.7 (C-3"'), 72.4 (C-4"”), 74.5 (C-2"), 76.2 (C-3”), 76.7 (C-5”), 93.5 (C8), 99.2 (C-6), 101.8 (C-1"), 101.2 (C-1"'), 104.5 (C10), 115.8 (C-2'), 116.7 (C-5'), 121.5 (C-6’), 121.8 (C1'), 133.8 (C-3), 145.2 (C-3'), 148.3 (C-4'), 156.4 (C-9), 156.6 (C-2), 161.8 (C-5), 164.8 (C-7), 177.8 (C-4). After comparing the structure of compound 1 with the literature $^{13}$, it was identified as rutin. 


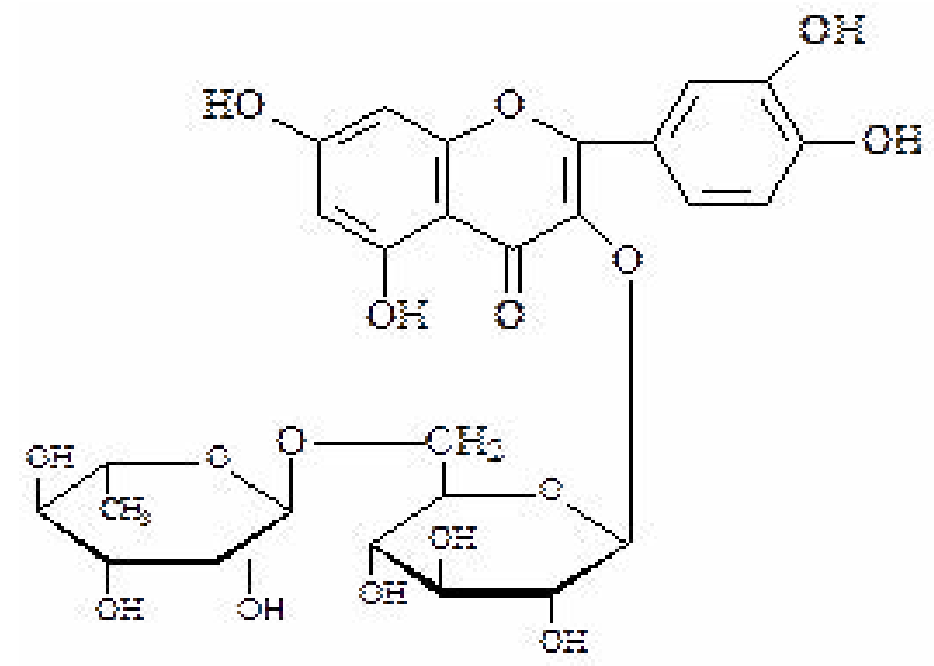

\section{Compound 2}

White needle crystals, $1 \mathrm{H}-\mathrm{NMR}(300 \mathrm{MHZ}) \delta: 0.62(3 \mathrm{H}$, s, H-23), 0.73 (3H, s, H-24), 0.89 (3H, s, H-25), 0.92 (3H, s, H-26), 0.84 (3H, s, H-29), 0.83 (3H, s, H-30), $1.12(3 \mathrm{H}, \mathrm{s}, \mathrm{H}-27), 3.02$ (1H, br.s, 3-OH), $4.25(1 \mathrm{H}, \mathrm{m}$, H-3), 5.18 (1H, br.s, H-12); 13C-NMR (ppm) $\delta: 15.8$ (C-25), 16.5 (C-24), 17.4 (C-26), 18.3 (C-6), 22.3 (C-11),
23.5 (C-16), 23.8 (C-30), 25.4 (C-27), 27.4 (C-2), 27.7 (C-15), 28.8 (C-23), 30.4 (C-20), 32.2 (C-7), 32.3 (C-29), 32.4 (C-21), 33.7 (C-21), 36.5 (C-10), 38.5 (C-1), 38.8 (C-4), 39.8 (C-8), 41.1 (C-14), 41.5 (C-18), 45.1 (C-19), 45.2 (C-17), 47.9 (C-9), 54.2 (C-5), 77.4 (C-3), 121.1 (C12), 144.3 (C-13), 178.5 (C-28). After comparing with the literature ${ }^{14}$, the structure of compound 2 was identified as oleanolic acid.

Figure 2: Oleanolic acid

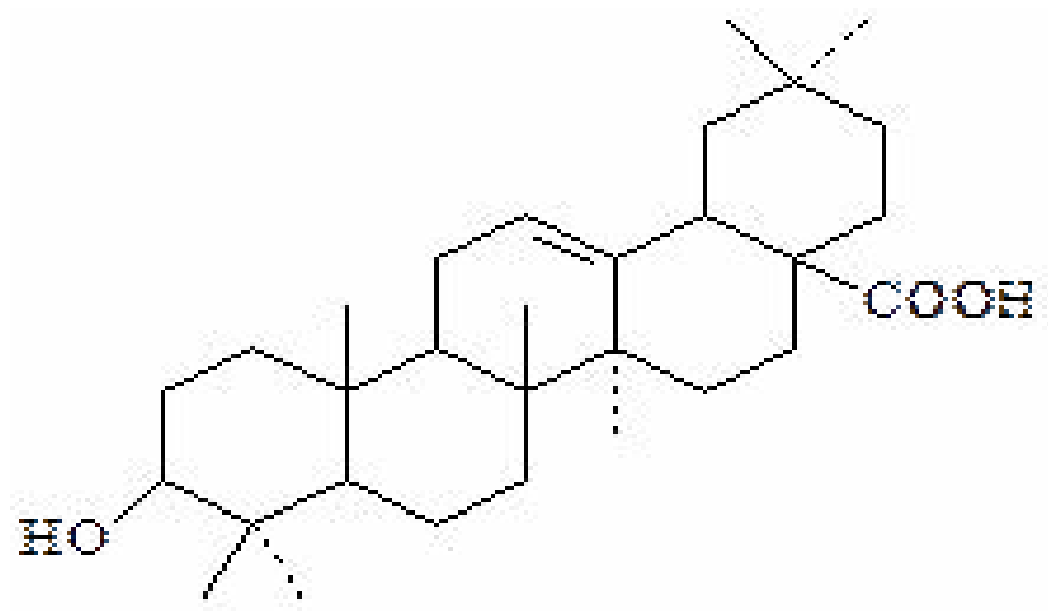

\section{Compound 3}

Yellowish-white powder. 1H-NMR (300MHZ) $8: 0.84$ (3H, t, H-11), 1.28 (2H, q, H-10), 1.53 (2H, t, H-9), 1.97 (2H, m, H-2), 2.11 (2H, m, H-6), 3.54 (1H, m, H-4), 3.85 (1H, m, H-5), 4.03 (2H, t, H-8), 5.08 (1H, m, H-3), $6.14\left(1 \mathrm{H}, \mathrm{d}, \mathrm{H}-2\right.$ '), $6.75\left(1 \mathrm{H}, \mathrm{d}, \mathrm{H}-8^{\prime}\right), 7.02(1 \mathrm{H}$, br.s, H-9'), 7.08 (1H, brs, H5'), 7.42 (1H, d, H-3'); 13C-NMR (ppm) 8: 173.5 (C-7), 165.7 (C-1'), 148.2 (C-7’), 145.4 (C-3'), 145.3 (C-6'), 125.8 (C-4'), 121.8 (C-9'), 116.2 (C-8'), 114.8 (C-2'), 114.3 (C-5'), 73.5 (C-1), 71.4 (C-4), 69.8 (C-3), 67.2 (C-5), 64.8 (C-8), 37.9 (C-2), 35.7 (C6), 30.5 (C-9), 18.2 (C-10), 13.9 (C-11). After identification $^{15}$, compound 3 was confirmed as chlorogenic acid butyl ester. 


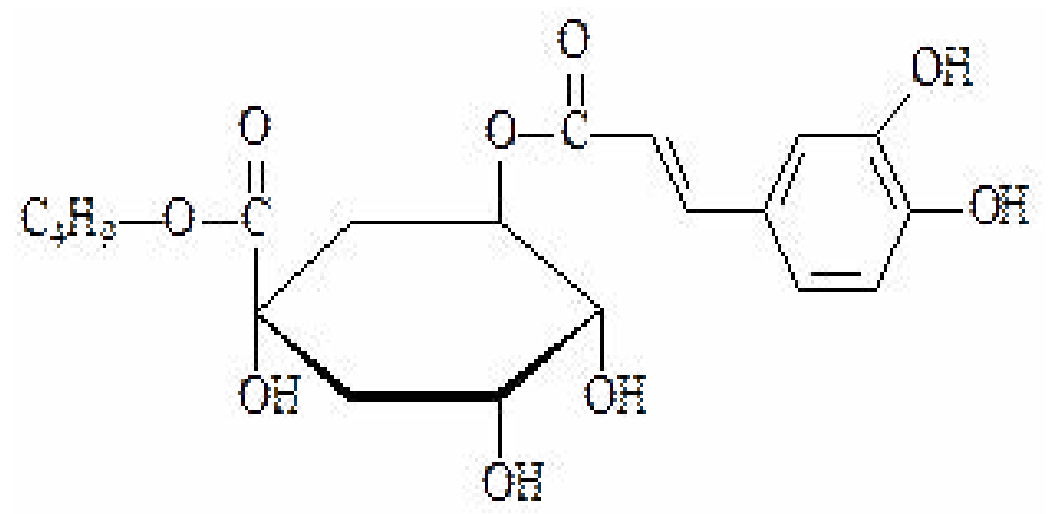

\section{Compound 4}

Yellow powder, positive to hydrochloric acid-magnesium reaction, upon chromatography on the same thin layer with quercetin reference substance using three different development systems, Rf values were all identical, suggesting that compound 4 was quercetin.

\section{Figure 4: Quercetin}<smiles>O=c1c(O)c(-c2ccc(O)c(O)c2)oc2cc(O)cc(O)c12</smiles>

Anti-inflammatory results of Lonicera japonica decoction

Effect of Lonicera japonica decoction on toe edema in rats

Results: as seen in Tab. 1, after injection of egg white, toe edema in rats in the control group was very obvious, different test concentrations of Lonicera japonica decoction all inhibited toe edema in rats to some extents, the edema was the mildest in the Lonicera japonica decoction high-dose group, which had the strongest inhibitory effect on the development of inflammation. Experimental results showed that at test concentrations, Lonicera japonica decoction had certain dose-effect relationship with toe edema in rats.

Table 1: Effect of Lonicera japonica decoction on toe edema in rats ( $\mathrm{mm}, \overline{\mathbf{x}} \pm \mathbf{s})$

\begin{tabular}{|c|c|c|c|c|}
\hline Group & $1 \mathrm{~h}$ & $2 \mathrm{~h}$ & $3 \mathrm{~h}$ & $4 \mathrm{~h}$ \\
\hline Control group & $1.65 \pm 0.23$ & $2.41 \pm 0.47$ & $2.72 \pm 0.45$ & $2.88 \pm 0.28$ \\
\hline Lonicera japonica decoction (high) & $\begin{array}{l}1.21 \pm \\
0.42 * *\end{array}$ & $\begin{array}{l}1.18 \pm \\
0.26^{* *}\end{array}$ & $\begin{array}{l}1.69 \pm \\
0.17 * *\end{array}$ & $\begin{array}{l}2.19 \pm \\
0.34 * *\end{array}$ \\
\hline $\begin{array}{l}\begin{array}{l}\text { Lonicera } \\
\text { (medium) }\end{array} \\
\text { japonica }\end{array}$ & $1.47 \pm 0.27 *$ & $1.69 \pm 0.22 *$ & $2.14 \pm 0.29 *$ & $2.37 \pm 0.26^{*}$ \\
\hline Lonicera japonica decoction (low) & $1.53 \pm 0.28$ & $1.89 \pm 0.53$ & $2.31 \pm 0.17$ & $2.55 \pm 0.33$ \\
\hline
\end{tabular}

Note: Comparison with the control group, ${ }^{*} \mathrm{P}<0.05$, ** $\mathrm{P}<0.01$ 
Effect of Lonicera japonica decoction on body temperature in rats

The results revealed that compared with the blank control group, the aspirin group and three dose groups of Lonicera japonica decoction could all lower the body temperature in rats, $3 \mathrm{~h}$ measurement results showed that the body temperature of rats in the blank group had already risen, while other groups were still able to lower the body temperature of rats under the action of test drugs. $4 \mathrm{~h}$ later, body temperature of rats in each group all went up, yet the elevation of body temperature was the fastest in the blank control group, indicating that compared with the blank control group, Lonicera japonica decoction could effectively reduce the body temperature of rats, this had an antipyretic effect.

\section{Fig. 5 Effect of Lonicera japonica decoction on body temperature in rats}

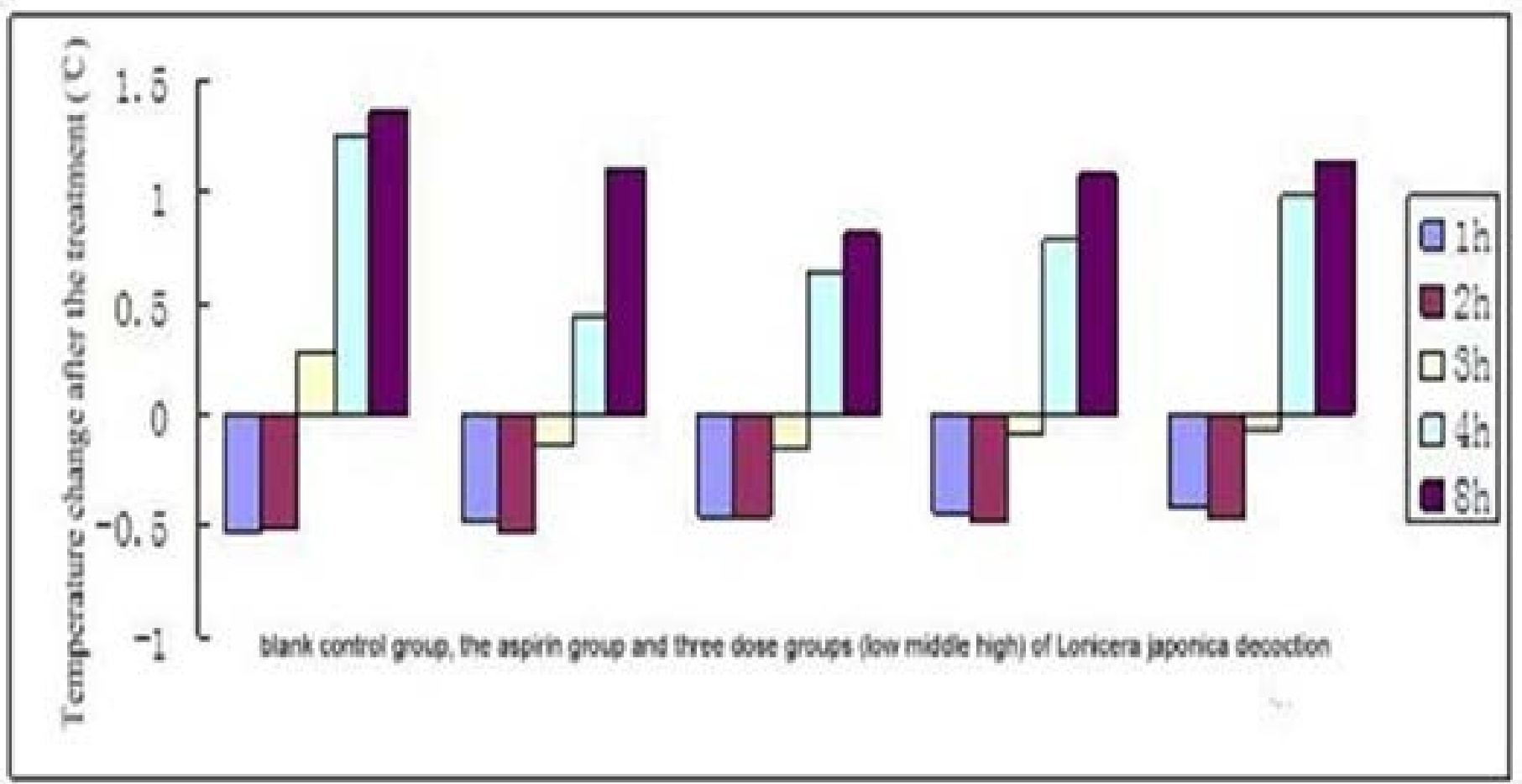

Anti-inflammatory effect of Lonicera japonica The severity of ear edema in mice in the blank control group was obvious, with increased thickness which showed significant difference between left and right ears. Under test doses, three Lonicera japonica decoc- tion inhibited xylene-inducedear edema in test groups of the mice, the inhibitory effect was especially significant in the high-dose group $(\mathrm{P}<0.01)$, suggesting that Lonicera japonica decoction had varying degrees of anti-inflammatory effects. See Fig. 6.

Fig. 6 Effect of Lonicera japonica decoction on ear edema in mice

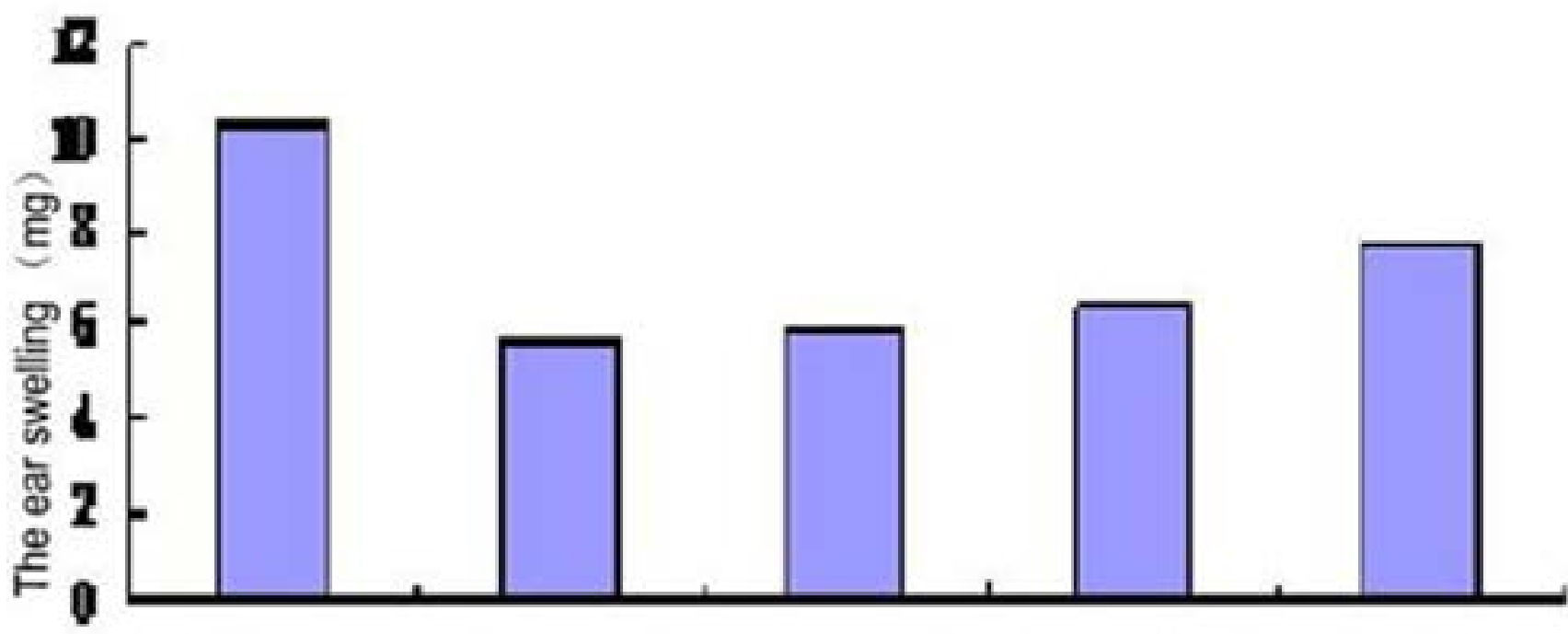




\section{Discussion}

As an important common traditional Chinese medicine, Lonicera japonica has been gaining much attention from physicians over the ages. It was listed as top grade in the "Supplementary Records of Famous Physicians". Famous doctor Li Shizhen described Lonicera japonica as "twining vine in trees, with purplish stems, and opposite leaves. Leaves are similar to Ficus pumila, green, with hair. Flowers bloom in March and April, long in one cun, with two flowers and two petals in a pedicle, one big one small, and long buds. The flowers open white then turn yellow two or three days later, together creating yellow and white colors, so is named Jin Yin Hua".

In this experiment, the ethanol extractum of Lonicera japonica was extracted successively with petroleum ether, ethyl acetate and n-butanol three times, respectively, extractants were evaporated, then the petroleum ether and ethyl acetate extracts were taken and subjected separately to silica gel column chromatography, and gradient-eluted with petroleum ether-acetone or chloroform-methanol system as eluent, after repeated silica gel column chromatography, ODS column chromatography and preparative chromatography, four compounds were isolated, namely quercetin, chlorogenic acid butyl ester, oleanolic acid and rutin.

Studies have shown that Lonicera japonica has anti-inflammatory and antibacterial effects, scholars have published a lot of studies and reports. But whether Lonicera japonica has an effect on pediatric respiratory tract infections, especially on upper respiratory infections has not been adequately reported, and its efficacy and mechanism of action are rarely reported.

Pediatric respiratory tract infections are one of the most common diseases of acute onset in children, common symptoms are manifested as fever, aversion to cold, nasal congestion, runny nose and sneezing, most are complicated by cough, and may accompany vomiting, diarrhea,or febrile seizures. The diseases have a high incidence, long duration, and are mostly seasonal, and especially epidemic at the turn of winter and spring. In the preliminary phase of the present study, 286 cases of pediatric upper respiratory tract infections over two years were collected, the children were aged between 8-14 years, with 148 boys, and 138 girls. During the process of treatment, in order to verify the therapeutic level of Lonicera japonica on upper respiratory tract in- fection in children, the children were randomly divided into two groups, namely the experimental group and the control group, the control group was given chemical medicine (acetaminophen medicine for cold), the experiment is published in another paper. The results showed that compared with the control group, the cure rate and average course of treatment were similar in the 143 cases of children who have taken Lonicera japonica decoction, suggesting significant therapeutic effect of Lonicera japonica decoction on upper respiratory tract infection in children.

During the process of inflammation, free radicals can be produced, adequate amount of free radicals can kill and remove harmful substances, but excessive, highly active oxygen radicals can easily damage the structure and function of cell membranes, resulting in the release of some active intracellular enzymes into the blood, and even lead to inflammatory edema ${ }^{16}$.

\section{Conclusion}

On the basis of the clinical application, the present experiment studied the anti-inflammatory effect of Lonicera japonica decoction. Experimental results showed that after injection of egg white, toe edema in rats in the control group was very obvious, different test concentrations of Lonicera japonica decoction all inhibited toe edema in rats to some extents, the edema was the mildest in the Lonicera japonica decoction high-dose group, which had the strongest inhibitory effect on the development of inflammation, the Lonicera japonica decoction showed certain dose-effect relationship with toe edema in rats. In the rat body temperature control experiment, while body temperature of rats in the blank group had already risen, other groups were still able to lower the body temperature of rats under the action of test drugs. The severity of ear edema in mice in the blank control group was obvious, with increased thickness which showed significant difference between left and right ears. Under test doses, three Lonicera japonica decoction groups all inhibited xylene-induced ear edema in mice, the above tests indicate that Lonicera japonica decoction has some anti-inflammatory effect, which will further explain the inhibition of pediatric upper respiratory tract infection by Lonicera japonica decoction.

\section{References}

1. Xiao PG. Modern Chinese Material Medica. Chemical Industry Press. 2002 edition; 736-737. 
2. Zhang L, Peng GF, Zhong FX, Lin HB, Wang XM. Analysis of chemical constituents in Shandong honeysuckle. Lishizhen Medicine and Materia Medica Research, 1996; 7(2): 89-91.

3. Gao YM, Wang MZ, Wang JM, Zhan Q, Qin HY, Mu HJ, Guan GJ, Wang H. Study of chemical constituents in Lonicera japonica. Chinese Traditional and Herbal Drugs, 1995; 26(11): 568-570.

4. Huang X, Li SL, Li P, Li HJ, Chai XY, Song Y. Simultaneous determination of eight main flavonoids in Flos lonicerae by high performance liquid chromatography. Acta Pharmacentica Sinica, 2005; 40(3): 285-288.

5. Lou HX, Lang WJ, Lv MJ. Isolation and structure determination of water-soluble compounds in Lonicera japonica. Chinese Traditional and Herbal Drugs, 1996; 27(4): 195-199.

6. Song Y, Li SL, Wu MH, Li HJ, Li P. Qualitative andquantitative analysis of iridoid glycosides in the flower buds of Lonicera species by capillary high performance liquid chromatography coupled with mass spectrometric detector. ELSEVIER, 2006: 564: 211-218 PubMed . 7. Wang TZ, Li YM, Wang ZX. Study on the volatile oil constituents in the buds of Lonicera macranthoides. Chinese Traditional and Herbal Drugs, 2000: 31(9): 657-658.

8. Fan HW, Xiao DW, Yu L, Zhu Q. The in vitro antiplatelet aggregative effects of Honeysuckle and its organic acid compounds. Chinese Journal of Hospital Pharmacy, 2006; 26(2): 145-147.
9. Huang XR, Liu WN, Cao D. Comments on Study of Pharmacological Effects of Chemical Components of Flos Lonicerae. Chinese Archives of Traditional Chinese Medicine, 2005; 23(3): 418-419.

10. Ge B, Lu XY, Yi K. The Active Constituent and Pharmaceutical Action of Flos Lonicerae and Its Application. Chinese Wild Plant Resources, 2004; 23(5): 13-16.

11. Li F, Li HQ. The study of Lonicera extract from water solution on ovalbumin-induced allergic mice model. Journal of Chongqing Medical University, 2004; 29(3): 288-291.

12. Ma YF. Study on Antioxidative Effect of Lonicera Similis Hemsl. Journal of Anbui Agricultural Sciences, 2007; 35(11): 3241-3242.

13. Chai XY, Li P, Tang LY. Studies on chemical constituents in dried buds of Lonicera confusa. China Journal of Chinese Materia Medica, 2004; 29(9): 865-867.

14. Wang QZ, Liang JY, Yuan Y. Chemical Constituents of Corydalis saxicola. Chinese Journal of Natural Medicines, 2007; 1: 31-34.

15. Sanehez AR. Vazquez P. Quinic acid esters from Isertia haenkeana. Photochemistry, 1990; 30(11): 311 PubMed -313.

16. Halliwell B. Oxidative stress, nutrition and health. Experimental strategies for optimization of nutritional antioxidant intake in humans. Free Radical Research, 1996; 25(1): 57-74. 